\title{
Methane-air flame thermometry using planar lase-induced fluorescence (PLIF)
}

\author{
Victor Fabelinsky ${ }^{1}$, Vitaly Kobtsev ${ }^{2 *}$, Sergey Kostritsa ${ }^{2}$, Dimitrii Kozlov ${ }^{1,2}$, \\ Valerii Smirnov ${ }^{1,2}$, and Konstantin Vereschagin ${ }^{1,2}$ \\ ${ }^{1}$ A.M. Prokhorov General Physics Institute, Russian Academy of Sciences, 119991 Moscow, Russia \\ ${ }^{2}$ Central Institute of Aviation Motors, 111116 Moscow, Russia
}

\begin{abstract}
Temperature measurements were performed in a central crosssection of a partially premixed atmospheric methane-air flame of a laboratory burner using two-line planar laser-induced fluorescence (PLIF) of $\mathrm{OH}$ radicals and coherent anti-Stokes Raman scattering (CARS) by $\mathrm{H}_{2}$ molecules. The results of a comparative analysis of 2D PLIF and singlepoint CARS thermometry of the flame are presented.
\end{abstract}

\section{Introduction}

Methods of linear and nonlinear laser spectroscopy are widely used in combustion research for aerospace engineering [1] to measure gas temperature and species concentration. In particular, the planar laser-induced fluorescence (PLIF) technique was applied [2-4] to hydrogen-containing flame diagnostics for detection of the hydroxyl $(\mathrm{OH})$ radical concentration and temperature spatial distributions. These data allow a more detailed flame characterization and can be used for verification of the combustion models.

Strong UV rovibronic transitions of the $\mathrm{OH}$ molecule $\mathrm{A}^{2} \Sigma^{+}<-\mathrm{X}^{2} \Pi$ bands provide a possibility to excite and detect 2D PLIF signals in the range with no IR- and visible flame radiation. On the other hand, CARS spectroscopy enables precise high spatial resolution single-point measurements. Therefore, PLIF and CARS techniques complement each other in flame diagnostics.

The present work is devoted to the experimental realization of spatially-overlapped registration of 2D PLIF images of $\mathrm{OH}$ radicals, excited at two wavelengths, and a set of single-point CARS-spectra of $\mathrm{H}_{2}$ molecules in a partially-premixed atmospheric methaneair flame of a laboratory burner, and to a comparative analysis of flame temperature distributions provided by these methods. The analysis is aimed to clarify the applicability of the two-line PLIF technique to thermometry of a spatially-inhomogeneous gas flow in a flame.

\footnotetext{
* Corresponding author: kobtsev.vitaly@gmail.com
} 


\section{Experimental}

The measurements were performed using a laboratory setup that includes the PLIF and CARS diagnostic systems, and a slot Wolfhard-Parker burner, with a "flat" flow of a combustible mixture in the center and a co-flow of air from aside, similar to that described in [5]. PLIF of $\mathrm{OH}$ intensity distributions, at two excitation wavelengths, and single-point CARS-spectra of $\mathrm{H}_{2}$ molecules were being recorded in the same central cross-section of a stationary partially-premixed atmospheric methane-air flame of the burner. This crosssection is perpendicular to the flame symmetry plane, i.e. to the slot of the burner.

The PLIF system consists of a tunable narrowband $\left(\approx 0.05 \mathrm{~cm}^{-1}\right) 10-\mathrm{ns} 8-\mathrm{Hz}$ frequencydoubled dye laser pumped by the second-harmonic radiation of a Nd:YAG laser, an optical system which forms a flat "laser knife", and an intensified CCD camera equipped with a UV objective and an interference (central wavelength $310 \mathrm{~nm}$, HWHM $10 \mathrm{~nm}$ ) and a colored glass short-pass (cut-off wavelength $350 \mathrm{~nm}$ ) filters which block the excitation and visible radiation. To obtain temperature distributions, PLIF signals were excited using two rovibronic transitions of the $A^{2} \Sigma^{+} \leftarrow X^{2} \Pi(1,0)$ band of $\mathrm{OH}$ radicals $\left(\mathrm{Q}_{1}(3)\right.$ $35420.38 \mathrm{~cm}^{-1}$, and $\left.\mathrm{Q}_{2}(10)-35121.08 \mathrm{~cm}^{-1}\right)$, with the energies of the lower states $E_{1}=202.38 \mathrm{~cm}^{-1}$ and $E_{2}=2056.55 \mathrm{~cm}^{-1}$, respectively. Images of PLIF in the $X^{2} \Pi \leftarrow A^{2} \Sigma^{+}(0,0)$ band for each of the excitation wavelengths, $I_{1}(m, n)$ and $I_{2}(m, n)$, where $m$ and $n$ are the image pixel coordinates, were obtained at about $1 \mathrm{~mJ}$ of the incident laser pulse energy as accumulation of 200 consecutive CCD frames.

If the excitation laser intensity distributions at two lines can be assumed to be homogeneous within the imaged spatial area, the ratio of the intensities in the PLIF images

$$
R(m, n)=I_{2}(m, n) / I_{1}(m, n)
$$

can be expressed as[3]

$$
R(m, n)=C \times \exp \left[-\left(E_{2}-E_{1}\right) /(k T(m, n))\right]
$$

Here, $k$ is the Boltzmann constant, $T(m, n)$ is local gas temperature, and $C$ is a coefficient. This coefficient depends on the ratios: of the absorption cross-sections of the involved transitions, of the excitation pulse energies and of the overlaps of the excitation and absorption line profiles, as well as of the parts of the excited $A^{2} \Sigma^{+}(v=1) \mathrm{OH}$ molecules transferred into the upper fluorescing $A^{2} \Sigma^{+}(v=0)$ state. If the assumption is valid that the coefficient $C$ weakly depends on gas temperature and composition, it can be derived experimentally by comparing the value of $R$ in a particular spatial point to the temperature in this point, independently determined using a reference technique. Further on, temperatures in all other spatial points can be calculated.

In this work, vibrational CARS thermometry was used as the reference technique to derive and compare the values of $C$ in a few spatial points, checking the validity of the assumption of its weak variation in the PLIF-probed cross-section of the flame, and to obtain 2D temperature distributions based on the PLIF signals in the areas where this assumption is valid. CARS-spectra of $\mathrm{H}_{2}$ molecules, which appear in the fuel-rich zones of the flame, were recorded using the CARS system employing broadband Stokes radiation and non-collinear configuration of crossing the beams. The detailed description of the system is given in [6]. The probe volume was $\sim 0.05 \mathrm{~mm}$ in diameter and $\sim 2 \mathrm{~mm}$ in length. CARS-spectra were recorded across the regions of the highest temperature gradients before the flame fronts at a few heights, $Z$, above the edge of the burner. 


\section{Results and discussion}

The atmospheric partially-premixed methane-air flame (Fig. 1, at the left) was operating at 5.9 slpm of methane-air mixture $(\Phi=2)$ and 22.3 slpm of dry air. PLIF signal distributions (Fig. 1, in the center) were recorded in the central cross-section of the flame.
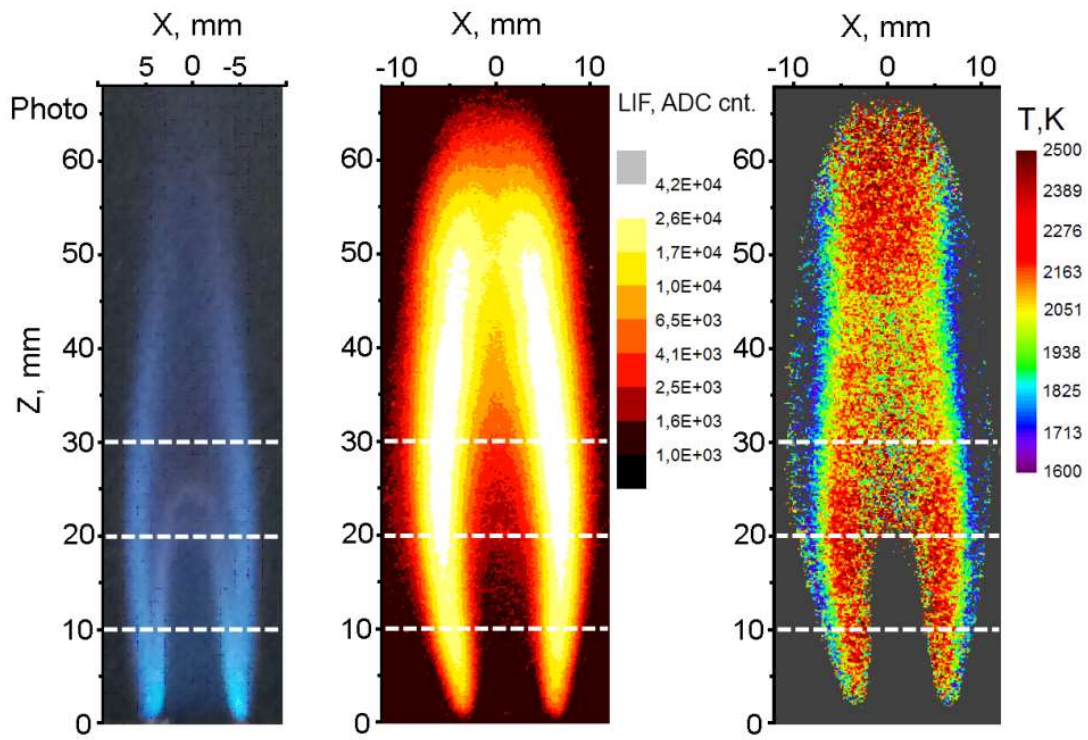

Fig. 1. A photograph of the partially-premixed fuel-rich atmospheric methane-air flame of the Wolfhard-Parker burner along the slot (at the left), a PLIF of OH intensity distribution excited via the $\mathrm{Q}_{2}$ (10) transition (in the center), and the calculated temperature distribution in the central crosssection of the flame (at the right) on the same scale; $X$ is the distance from the flame symmetry plane. The dashed lines show the heights above the edge of the burner at which CARS temperature measurements involved in the derivation of the coefficient $C$ were performed.

CARS-spectra of $\mathrm{H}_{2}$, recorded in the same cross-section at the heights 10, 20, and $30 \mathrm{~mm}$ above the edge of the burner with steps of 1-2 mm, were processed to derive local gas temperatures. The obtained values are in a reasonable agreement with those derived from vibrational CARS-spectra of $\mathrm{N}_{2}$ in the similar points of the methane-air flame of a similar Wolfhard-Parker burner run at the equivalent flow conditions [7].

The values of the coefficient $C$ of correspondence between the ratios of PLIF intensities and the temperatures were calculated for the points of CARS measurements. In the significant part of the probed flame cross-section area, where $\mathrm{OH}$ radicals exist and fluoresce, the spread of the derived $C$ values was found to be less than $10 \%$. This confirmed the validity of the assumption of the weak dependence of $\boldsymbol{C}$ on temperature and species concentration for the considered part of the cross-section and allowed us to use the mean value of $C$ for the subsequent calculation of the $2 \mathrm{D}$ temperature distribution (Fig. 1, in the right). For this, the PLIF signal cut-off level was chosen to be $5 \%$ of the maximal value. This selection was conditioned by the amplitude of noise of the PLIF signals resulting in significant errors in derived temperatures if the values below this level were taken.

Figs. 2 and 3 represent spatially averaged over $2 \mathrm{~mm}$ in height profiles of the calculated 2D temperature distribution, together with CARS temperature data, obtained in the present work and in [7], at the heights of 10 and $30 \mathrm{~mm}$ above the burner edge. The profiles of the PLIF signal distribution in Fig. 1, at the same heights, are also presented. The spatial filtering of the PLIF signals is required to obtain smooth temperature distributions at the relatively high spread of the derived values. As a result, the temperature measurements 
using PLIF demonstrate lower spatial resolution than those employing CARS, but provide larger field of data for a shorter time.

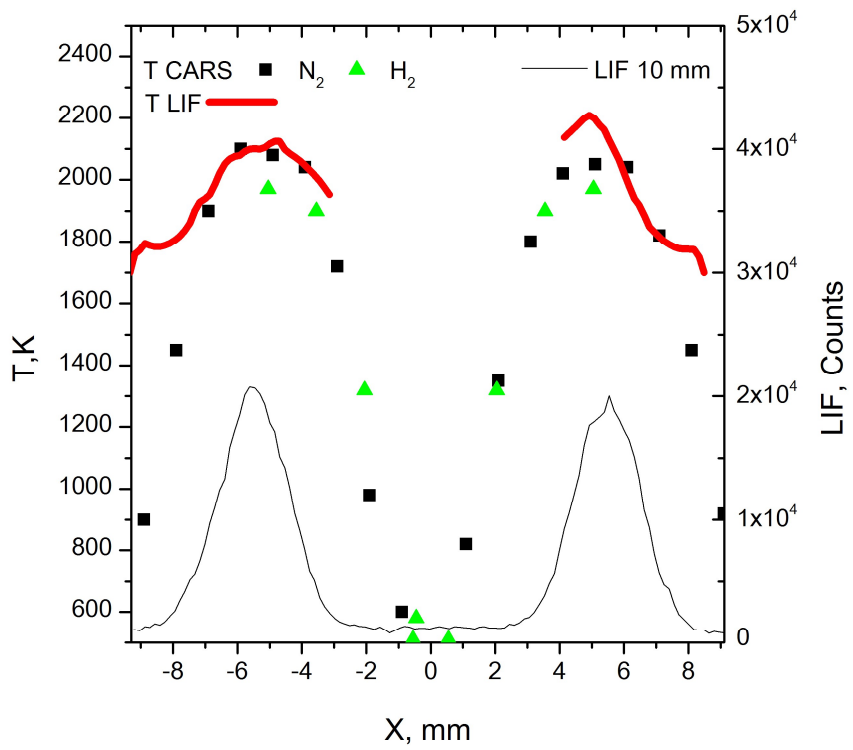

Fig. 2. Calculated temperature profiles (smoothed OH PLIF measurements - bold red line, CARS by $\mathrm{H}_{2}$ - green triangles, CARS by $\mathrm{N}_{2}$ [7] - black squares) and PLIF intensity profile (thin black line) at $10 \mathrm{~mm}$ above the burner edge.

The defined spread of $C$ values does not result in significant deviations between the PLIF and CARS temperature profiles (less than $150 \mathrm{~K}$ ), and the values provided by the techniques are in a reasonable agreement. Note, that the PLIF signals are not intense enough for the calculations in the low-temperature regions $(\mathrm{T}<1600 \mathrm{~K})$ - in the central part of the combustible mixture flow, at the heights below $20 \mathrm{~mm}$ (the inner flame front, Fig, 1), and in the outer part of the flame.

\section{Conclusion}

Complementary measurements were carried out in the central section of the partiallypremixed atmospheric methane-air flame of the laboratory slot burner with spatiallyinhomogeneous species and temperature profiles using two-line PLIF of OH radicals and CARS by $\mathrm{H}_{2}$ molecules.

Processing of the PLIF images registered at two excitation wavelengths employing the results of CARS temperature measurements allowed us to derive the values of the correspondence coefficient $C$ between the ratios of PLIF intensities and the temperatures in the specific points of the PLIF images.

Small spread of the derived $C$ values was obtained in the significant part of the probed flame cross-section area, where $\mathrm{OH}$ radicals exist and fluoresce. This confirmed the validity of the assumption of the weak dependence of $C$ on temperature and species concentration for the considered part of the cross-section and allowed us to convert the two PLIF images into the $2 \mathrm{D}$ temperature field. The calculated temperatures were found to match the results of our $\mathrm{H}_{2}-\mathrm{CARS}$ measurements and $\mathrm{N}_{2}-\mathrm{CARS}$ data from the literature. 


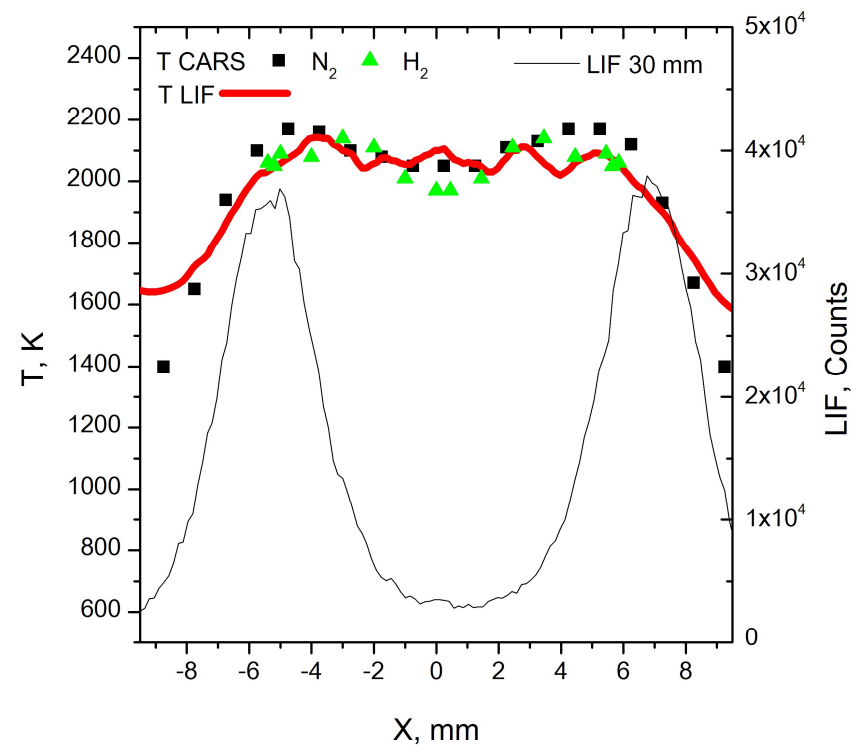

Fig. 3. Calculated temperature profiles (smoothed OH PLIF measurements - bold red line, CARS by $\mathrm{H}_{2}$ - green triangles, CARS by $\mathrm{N}_{2}$ [7] - black squares) and PLIF intensity profile (thin black line) at $30 \mathrm{~mm}$ above the burner edge.

The help of T. Seeger (University of Siegen, Germany) with manufacturing and tests of the WolfhardParker burner in frame of the Cooperation Program is gratefully acknowledged. The work was financially supported by CIAM Foundation for Priority Research.

\section{References}

1. A. Ehn, J. Zhu, X. Li, and J. Kiefer, Appl. Spectrosc. 71 (5), 1-26, (2017)

2. R.K. Hanson, K. Ronald, J. M. Seitzman, P. H. Paul. Appl. Phys. B, 50 (6), 441-454 (1990)

3. J. M. Seitzman, R. K. Hanson, P. A. DeBarber, C. F. Hess, Applied Optics, 33 (18), 4000-4012 (1994)

4. U. E. Meier, D. Wolff-Gaßmann, W. Stricker, Aerosp. Sci. and Tech. 4 (6), 403 - 414 (2000)

5. K.C. Smyth, J.H. Miller, R.C. Dorfman, W.G. Mallard, R.J. Santoro Combust. Flame, 62, 157-181 (1985)

6. V.V. Smirnov, S.A. Kostritsa, V.D. Kobtsev, N.S. Titova, A.M. Starik, Combust. Flame, 162 (10), 3554-3561 (2015)

7. A. Datta, F. Beyrau, T. Seeger, A. Leipertz, Combust. Sci. and Techn. 176, 1-20 (2004) 